\title{
Second-harmonic generation by a graphene nanoparticle
}

\author{
Daria A. Smirnova, ${ }^{1}$ Ilya V. Shadrivov, ${ }^{1}$ Andrey E. Miroshnichenko, ${ }^{1}$ Alexander I. Smirnov, ${ }^{2,3}$ and Yuri S. Kivshar ${ }^{1}$ \\ ${ }^{1}$ Nonlinear Physics Center, Research School of Physics and Engineering, Australian National University, Canberra ACT 0200, Australia \\ ${ }^{2}$ Lobachevsky State University, Nizhny Novgorod 603950, Russia \\ ${ }^{3}$ Institute of Applied Physics, Russian Academy of Sciences, Nizhny Novgorod 603950, Russia
}

(Received 6 May 2014; revised manuscript received 10 June 2014; published 11 July 2014)

\begin{abstract}
We study the second-harmonic generation by a spherical dielectric nanoparticle covered by graphene. We demonstrate that a strong nonlinear response is caused by an induced surface current in the graphene nanoparticle illuminated by an external electromagnetic wave. We obtain analytical expressions for the field multipoles characterizing the second-harmonic radiation and analyze the dependence of intensity and directivity of the nonlinear scattering on the frequency and structure of the electromagnetic field, revealing the asymmetric radiation patterns due to constructive multipole interference for the resonantly enhanced second-harmonic generation.
\end{abstract}

DOI: 10.1103/PhysRevB.90.035412 PACS number(s): 42.65.Wi, 78.67.Wj, 73.25.+i, 78.68.+m

\section{INTRODUCTION}

Nonlinear optical properties of metallic thin films and plasmonic nanoparticles were the subject of extensive theoretical and experimental studies for several decades [1-9], and these studies laid the foundation of nonlinear plasmonics [10]. The studies of nonlinear effects accompanying the propagation and scattering of light in nanostructured media with metal inclusions was stimulated by rapid progress in nanofabrication technology and a growing interest in optical metamaterials [11].

Second-harmonic generation (SHG) of optical radiation by individual metal nanoparticles has been discussed in several papers [12-22]. In particular, it was shown that dipole and quadrupole electrical moments excited in the particle at the second harmonic frequency provide the main contribution to the radiation, and they can be expressed via the bulk and surface susceptibility tensors of the second order [13,17]. More recently, the idea of all-optical control of the radiation directionality through an interplay between linear light scattering and second-harmonic generation was proposed in the context of nanoantennas [23].

Electromagnetic properties of structures containing graphene attracted significant attention in past years leading to the rapid development of a new branch of plasmonics, which is now widely called graphene nanoplasmonics [24-28]. Graphene is suggested as an alternative to conventional metalbased structures to confine light and guide surface plasmon polaritons [29,30]. Studies of nonlinear optical properties of graphene are still at relatively early stages, with several theoretical and experimental works published to date [31-35]. Several nonlinear effects have been studied and demonstrated for the structures with graphene, including the theoretical prediction of spatial plasmon solitons supported by the selfaction effect of light in graphene [36-38].

Importantly, graphene is not only employed for planar geometries, and there are also attempts to create graphenewrapped objects [39-41]. Further theoretical studies suggest that by wrapping spherical nanoparticles in graphene it becomes possible to achieve tunable cloaking effect [42-44].

In this paper we study a nonlinear response of a graphenewrapped dielectric spherical nanoparticle. We consider a nanoparticle with an outer graphene layer and analyze the second-harmonic generation induced by the nonlinear conductivity of graphene. We develop a theoretical model which allows one to obtain analytical expressions for the multipole decomposition of the second-harmonic scattered field. We study second-harmonic radiation patterns in such a structure and explore the possibilities to control the emission profile by inhomogeneous fields.

Since a graphene layer is many orders of magnitude thinner than the wavelength of the electromagnetic waves under consideration, graphene can be treated as a conductive surface described by the Dirac $\delta$ function [45]. The frequencydependent surface conductivity of graphene has been derived in a number of works [46-48], and we use the result of Ref. [48] written in the form

$$
\begin{aligned}
\sigma_{s}(\omega) & =\sigma_{\text {intra }}(\omega)+\sigma_{\text {inter }}(\omega) \\
& =\frac{i e^{2}}{\pi \hbar}\left[\frac{\mu}{\hbar\left(\omega+i \tau_{\text {intra }}^{-1}\right)}+\frac{1}{4} \ln \left|\frac{2 \mu-\hbar \omega}{2 \mu+\hbar \omega}\right|\right],
\end{aligned}
$$

where we assume for doped graphene $\hbar \omega<1.67 \mu$ and $k_{B} T \ll$ $\mu$, where $\mu$ is the chemical potential, $k_{B}$ is the Boltzmann constant, $T$ is the temperature, and $\tau_{\text {intra }}^{-1}$ is the relaxation rate. In the semiclassical limit $\hbar \omega \leqslant \mu$ the linear conductivity of graphene can be reduced to the Drude form $\sigma_{s}(\omega) \approx \sigma_{\text {intra }}(\omega)$, which takes into account only intraband processes $[31,48]$.

We note that for a randomly stacked multilayer graphene film consisting of $N$ layers, at low frequencies the equivalent surface conductivity is $N$ times larger than that of a monolayer graphene [38,49-53], i.e., $\sigma(\omega)=N \sigma_{s}(\omega)$. This allows us to engineer the conductivity of graphene-based structures. Importantly, the increase of the effective conductivity with a number of layers leads to a substantial reduction of the wave number of the $p$-polarized plasmons supported by multilayer graphene structures. This provides a mechanism for efficient control of both the plasmon localization and dispersion which is important for real nanophotonic applications [38,53]. In what follows we develop the theoretical approach which is applicable to both mono- and multilayer graphene structures.

\section{MODEL}

In the first part of the paper, which includes Sections II-IV, we consider the Rayleigh scattering of a 


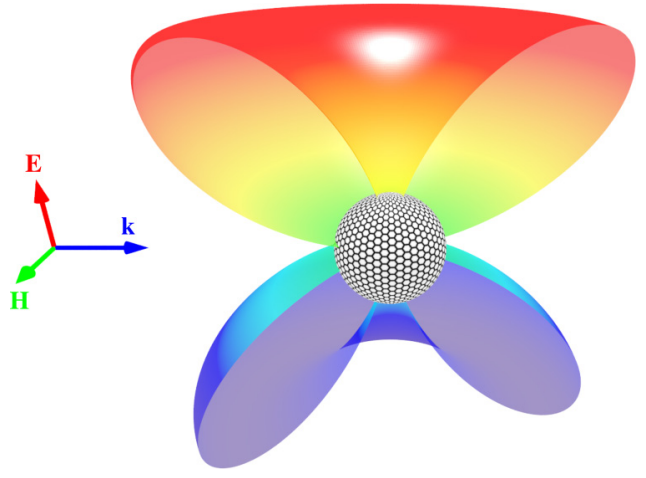

FIG. 1. (Color online) Schematic view of the graphene-wrapped nanoparticle illuminated by a plane wave. Surface plot shows a predominantly quadrupole second-harmonic radiation pattern formed in the far field when the fundamental frequency is close to the one-photon dipolar resonance.

linearly polarized plane monochromatic electromagnetic wave $\left[\mathbf{E}=E_{0} \mathbf{z}_{0} \exp \left(-i \omega_{0} t+i k_{0} \sqrt{\varepsilon_{h}} y\right)\right]$ on a graphene sphere of radius $a \ll \lambda_{0}=2 \pi / k_{0} \sqrt{\varepsilon_{h}}, k_{0}=\omega_{0} / c$ (see schematics in Fig. 1).

The incident wave excites the fundamental frequency harmonics whose electric field is governed by the equation

$$
-\nabla \times \nabla \times \mathbf{E}_{\omega}+\frac{\omega^{2}}{c^{2}} \varepsilon(\mathbf{r}) \mathbf{E}_{\omega}=-\frac{4 \pi i \omega}{c^{2}}\left(\mathbf{j}_{\omega}^{\mathrm{L}}+\mathbf{j}_{\omega}^{\mathrm{NL}}\right) \delta(r-a),
$$

where $\mathbf{j}_{\omega}^{\mathrm{L}}=\sigma(\omega) \mathbf{E}_{\tau}$ and $\mathbf{j}_{\omega}^{\mathrm{NL}}$ are the surface densities of the linear and nonlinear currents at the frequency $\omega$, respectively, and subscript $\tau$ refers to the field component tangential to the surface. Here, we introduced the dielectric permittivity function

$$
\varepsilon(\mathbf{r})= \begin{cases}\varepsilon_{p}, & |\mathbf{r}|<a \\ \varepsilon_{h}, & |\mathbf{r}|>a,\end{cases}
$$

which describes the dielectric permittivity distribution in space. This permittivity describes a dielectric particle located in a homogeneous host medium. In what follows, we assume that the particle is placed in vacuum, i.e., $\varepsilon_{h}=1$.

The approach we employ is based on the multipole expansion of the electromagnetic fields [54,55]. For describing nanoparticles this method is mainly associated with the Mie scattering theory and it gives a basic understanding of harmonic generation from nanoparticles as was discussed theoretically, e.g., by Dadap et al. $[13,17]$ for small spheres. In particular, previous studies show that the SHG response of a nanoparticle irradiated by a linearly polarized plane wave is dominated by an electric quadrupole and electric dipole originating from the finite size effect and from retardation effect. Remarkably, the magnetic dipole despite being of the same order as an electric quadrupole is not excited because of symmetry constraints. This will also be relevant in the problem that we consider in this paper. The strength of the excited leading multipoles and their interference determine far-field radiation characteristics, such as a radiation pattern and radiation efficiency.
In what follows we present a self-consistent derivation underlining the important specifics of graphene as a nonlinear material that, in some sense, may be regarded as a very peculiar model of the surface nonlinearity associated with Dirac particles. We would also like to note that though we solve a model problem in the simplest spherical geometry we believe that our findings can be useful in designing more complex shape graphene-based nanoantennas.

\section{LINEAR SCATTERING}

As follows from (2), in an approximation that is linear in $E_{\omega}$, outside and inside the sphere

$$
\nabla \cdot \mathbf{D}_{\omega}=0,
$$

where $\mathbf{D}_{\omega}=\varepsilon(\mathbf{r}) \mathbf{E}_{\omega}$ is the electric displacement vector. In the case when the radius $a$ of the particle is small, i.e., $k a \ll 1$ and $k \sqrt{\varepsilon_{p}} a \ll 1(k=\omega / c)$ are fulfilled, the solution can be found using a perturbation method with the small parameter $\mathcal{M}(\omega)=k a \equiv a \omega / c \ll 1$, assuming $\varepsilon_{p} \sim 1$. The first term in this expansion corresponds to the quasistatic approximation [54,56].

We represent the total field in the structure $\mathbf{E}_{\omega_{0}}$ as a superposition of the field of the incident wave $\mathbf{z}_{0} E_{0} e^{i k_{0} y}$ and the scattered field $\mathcal{E}_{\omega_{0}}$ :

$$
\mathbf{E}_{\omega_{0}}=\mathbf{z}_{0} E_{0} e^{i k_{0} y}+\mathcal{E}_{\omega_{0}} .
$$

Next, we represent the scattered field as an asymptotic series with the small parameter $\mathcal{M}_{0}=\mathcal{M}\left(\omega_{0}\right)$ in the region $k_{0} r \ll 1$ in the form

$$
\mathbf{E}_{\omega_{0}}=E_{0} \mathbf{z}_{0}+\mathcal{E}_{\omega_{0}}^{(0)}+\left(\mathcal{E}_{\omega_{0}}^{(1)}+i k_{0} y E_{0} \mathbf{z}_{0}\right)+\cdots,
$$

where the fields in the brackets are of the order of $\mathcal{M}_{0}$.

In the zeroth and first orders of smallness in $\mathcal{M}_{0}$ the scattered fields $\mathcal{E}_{\omega_{0}}^{(0)}$ and $\mathcal{E}_{\omega_{0}}^{(1)}$ are potential:

$$
\mathcal{E}_{\omega_{0}}^{(0,1)}=-\nabla \Phi_{\omega_{0}}^{(0,1)} .
$$

From Eq. (4) it follows that the potential functions $\Phi_{\omega_{0}}^{(0)}$ and $\Phi_{\omega_{0}}^{(1)}$ both outside and inside the sphere satisfy the Laplace equation

$$
\nabla^{2} \Phi_{\omega_{0}}^{(0,1)}=0 \quad(r<a, r>a) .
$$

The tangential electric field components, in general, can experience a discontinuity at the surface of the nanoparticle. This discontinuity is proportional to the surface gradient of the normal component of the current density. In our case of small energies near the $K$ point where transitions occur between the $\pi-\pi^{*}$ bonds, the normal component of the current is negligible. Therefore, the tangential component of the electric field is continuous at the boundary $r=a$, i.e., $\mathbf{E}_{\omega_{0}}^{(0)}=E_{0} \mathbf{z}_{0}-\nabla \Phi_{\omega_{0}}^{(0)}$ and $\mathbf{E}_{\omega_{0}}^{(1)}=i k_{0} y E_{0} \mathbf{z}_{0}-\nabla \Phi_{\omega_{0}}^{(1)}$ are continuous, whereas the normal components of the vectors $\mathbf{D}_{\omega_{0}}^{(0)}=\varepsilon(\mathbf{r})\left(-\nabla \Phi_{\omega_{0}}^{(0)}+\right.$ $\left.E_{0} \mathbf{z}_{0}\right)$ and $\mathbf{D}_{\omega_{0}}^{(1)}=\varepsilon(\mathbf{r})\left(-\nabla \Phi_{\omega_{0}}^{(1)}+i k_{0} y E_{0} \mathbf{z}_{0}\right)$ undergo jumps at the surface charges $\rho_{\omega_{0}}^{(0,1)}=\nabla_{s} \cdot \mathbf{j}_{\omega_{0}}^{\mathrm{L}(0,1)} / i \omega_{0}$, where the operator $\nabla_{s} \cdot$ stands for the surface divergence. The potentials $\Phi_{\omega_{0}}^{(0)}$ and $\Phi_{\omega_{0}}^{(1)}$, which satisfy such boundary conditions and 
decay away from the particle, are given by

$$
\begin{gathered}
\Phi_{\omega_{0}}^{(0)}=a^{3} E_{0} \cos \theta \frac{\varepsilon_{p}-1-2 \Theta_{1}}{\varepsilon_{p}+2-2 \Theta_{1}} \begin{cases}\frac{r}{a^{3}}, & |\mathbf{r}|<a \\
\frac{1}{r^{2}}, & |\mathbf{r}|>a,\end{cases} \\
\Phi_{\omega_{0}}^{(1)}=a^{5} E_{0} \sin 2 \theta \sin \varphi \frac{i k_{0}\left(\varepsilon_{p}-1-3 \Theta_{1}\right)}{4\left(\varepsilon_{p}+\frac{3}{2}-3 \Theta_{1}\right)} \begin{cases}\frac{r^{2}}{a^{5}}, & |\mathbf{r}|<a \\
\frac{1}{r^{3}}, & |\mathbf{r}|>a,\end{cases}
\end{gathered}
$$

where $\Theta_{1}=\frac{4 \pi \sigma_{1}}{i \omega_{0} a}, \sigma_{1} \equiv \sigma\left(\omega_{0}\right), \theta$ is the polar (zenith) angle measured from the $z$ axis, and $\varphi$ is the azimuthal angle measured from the $x$ axis in a spherical coordinate system with its origin in the center of a nanoparticle.

As seen from (9), the scattered field $\mathcal{E}_{\omega_{0}}$ in the zeroth and first orders of the perturbation theory with respect to the parameter $\mathcal{M}_{0}$ for $r>a$ is the superposition of the field of a point dipole having the dipole moment

$$
\mathcal{P}_{\omega_{0}}^{(0)}=\mathbf{z}_{0} E_{0} a^{3} \frac{\varepsilon_{p}-1-2 \Theta_{1}}{\varepsilon_{p}+2-2 \Theta_{1}},
$$

and the field of a quadrupole with the quadrupole moment tensor

$$
\hat{Q}_{\omega_{0}}^{(1)}=Q_{\omega_{0}}^{(1)}\left(\begin{array}{ccc}
0 & 0 & 0 \\
0 & 0 & 1 \\
0 & 1 & 0
\end{array}\right)
$$

with two nonzero components

$$
Q_{y z}=Q_{z y}=Q_{\omega_{0}}^{(1)}=E_{0} i k_{0} a^{5} \frac{\varepsilon_{p}-1-3 \Theta_{1}}{2 \varepsilon_{p}+3-6 \Theta_{1}} .
$$

\section{NONLINEAR SCATTERING AND SECOND-HARMONIC GENERATION}

Because of the graphene nonlinearity, the field $\mathbf{E}_{\omega_{0}}$ excites higher harmonics, and here we consider only the secondharmonic $\mathbf{E}_{2 \omega_{0}}$ generation. To describe nonlinear properties of graphene, we employ the quasiclassical description based on the solution of the Boltzmann equation, applicable at low frequencies, $\hbar \omega \leqslant \mu[31,32,35,57]$.

In the case of a plane graphene, in the collisionless limit, the electron distribution function $f(\mathbf{r}, \mathbf{p}, t)$ satisfies the kinetic equation written as

$$
\frac{\partial f}{\partial t}+\mathbf{v}_{\mathbf{p}} \frac{\partial f}{\partial \mathbf{r}}+e\left(\mathbf{E}+\frac{1}{c}\left[\mathbf{v}_{\mathbf{p}} \times \mathbf{B}\right]\right) \frac{\partial f}{\partial \mathbf{p}}=0,
$$

where for Dirac's massless quasiparticles $\mathbf{v}_{\mathbf{p}}=\frac{\partial \mathcal{E}}{\partial \mathbf{p}}=v_{F} \frac{\mathbf{p}}{|\mathbf{p}|}$, the energy $\mathcal{E}=v_{F} p, v_{F} \approx c / 300$ is the Fermi velocity, and $e=-|e|$ is the charge. Following the perturbation approach $[57,58]$, we solve this equation using iterations. The electric current density at $2 \omega$ is given through the second-order correction to the Fermi-Dirac distribution function $\mathbf{j}_{2 \omega}=$ $4 e \sum_{\mathbf{p}} \mathbf{v}_{\mathbf{p}} f_{2 \omega}(\mathbf{p})$.

We look for the distribution function in the form

$$
f=f_{0}(\varepsilon)+f_{\omega} e^{-i \omega t}+\text { c.c. }+f_{2 \omega} e^{-2 i \omega t}+\text { c.c. }+\cdots,
$$

where $f_{0}(\mathcal{E})$ is the Fermi-Dirac distribution, and $f_{\omega}$ and $f_{2 \omega}$ are the first- and the second-order corrections, respectively, whereas the tangential electric field

$$
\mathbf{E}_{\tau}=\mathbf{E}_{\omega} e^{-i \omega t}+\mathbf{E}_{2 \omega} e^{-2 i \omega t} \ldots
$$

Finally, under the assumption $v_{F} / \omega \ll l_{E}$, where $l_{E}$ is a characteristic spatial scale of the field inhomogeneity, we obtain the general expression for the current at the second harmonic,

$$
\begin{aligned}
\mathbf{j}_{2 \omega}= & \frac{2 e^{3} i}{\omega^{3}(2 \pi \hbar)^{2}} \iint d^{2} \mathbf{p} \mathbf{v}_{\mathbf{p}}\left[\left(\mathbf{E}_{\omega} \frac{\partial}{\partial \mathbf{p}}\right)\left(\mathbf{v}_{\mathbf{p}} \frac{\partial}{\partial \mathbf{r}}\right)\right. \\
& \left.\times\left(\mathbf{E}_{\omega} \frac{\partial f_{0}}{\partial \mathbf{p}}\right)+\frac{1}{2}\left(\mathbf{v}_{\mathbf{p}} \frac{\partial}{\partial \mathbf{r}}\right)\left(\mathbf{E}_{\omega} \frac{\partial}{\partial \mathbf{p}}\right)\left(\mathbf{E}_{\omega} \frac{\partial f_{0}}{\partial \mathbf{p}}\right)\right] .
\end{aligned}
$$

We note in the case of flat graphene and transversemagnetic-polarized incident wave with the tangential electric field component of the form $\mathbf{E}_{\omega}=E \mathbf{x}_{0} e^{i q x}$ (and $v_{F} / \omega \ll 1 / q$ ) Eq. (16) is reduced to

$$
\mathbf{j}_{2 \omega}=-\mathbf{x}_{0} \frac{3}{8} \frac{e^{3} v_{F}^{2}}{\pi \hbar^{2} \omega^{3}} q E^{2} e^{2 i q x},
$$

which is in agreement with the earlier results presented in Ref. [58].

We consider particles which are large enough on the scale of electron motion in graphene (i.e., $v_{F} / \omega \ll a$ and $v_{F} \tau_{\text {intra }} \ll$ $a)$, then at each point of the surface of the particle, the current can be considered locally flat. As a result, for a description of the second-harmonic current we can use Eq. (16), where $\mathbf{v}_{\mathbf{p}}$ and $\mathbf{p}$ are vectors tangential to the surface. Then, we find the resulting formula for the second-harmonic currents induced on the spherical object in the zeroth order of the small parameter $\mathcal{M}_{0}$, and it corresponds to the dipole field distribution

$$
\mathbf{j}_{2 \omega_{0}}^{(0)}=\boldsymbol{\theta}_{0} i \frac{3}{16} \frac{e^{3}}{\pi \hbar^{2} \omega_{0}^{3}} \frac{v_{F}^{2}}{a} E_{d}^{2} \sin 2 \theta .
$$

In the first order of small parameter $\mathcal{M}_{0}$ we find

$$
\begin{aligned}
\mathbf{j}_{2 \omega_{0}}^{(1)}= & \mathbf{j}_{2 \omega_{0 \mathrm{I}}}^{(1)}+\mathbf{j}_{2 \omega_{0 \mathrm{II}}}^{(1)}, \\
\mathbf{j}_{2 \omega_{0 \mathrm{I}}}^{(1)}= & i \frac{e^{3}}{\pi \hbar^{2} \omega_{0}^{3}} \frac{v_{F}^{2}}{a} E_{d} E_{q}\left[\boldsymbol{\theta}_{0} \sin \varphi\left(\frac{13}{16} \cos \theta-\frac{9}{16} \cos 3 \theta\right)\right. \\
& \left.+\boldsymbol{\varphi}_{0} \frac{1}{4} \cos 2 \theta \cos \varphi\right] \\
\mathbf{j}_{2 \omega_{0 \mathrm{II}}}^{(1)}= & i \frac{e^{3} v_{F}^{2}}{\pi \hbar^{2} \omega_{0}^{3}} E_{d}\left(\frac{-i k_{0} E_{0}}{8}\right) \\
& \times\left[\boldsymbol{\theta}_{0} \sin \varphi \cos \theta+\boldsymbol{\varphi}_{0} \frac{1}{2}(3-\cos 2 \theta) \cos \varphi\right]
\end{aligned}
$$

where the amplitude coefficients

$$
\begin{aligned}
E_{d} & =E_{0}\left(1+\frac{2 \Theta_{1}+1-\varepsilon_{p}}{\varepsilon_{p}+2-2 \Theta_{1}}\right) \\
& =E_{0} \frac{3}{\varepsilon_{p}+2-2 \Theta_{1}}, \\
E_{q} & =E_{0} \frac{2.5 i k_{0} a}{2 \varepsilon_{p}+3-6 \Theta_{1}} .
\end{aligned}
$$


For multilayer graphene wrappings, the obtained nonlinear currents should be multiplied by a number of layers.

Similar to how it is done in Sec. III for the fundamental frequency wave, here we represent the field of the second harmonics $\mathbf{E}_{2 \omega_{0}}$ inside and in the vicinity of a nanoparticle as an asymptotic series in the parameter $\mathcal{M}\left(2 \omega_{0}\right)=2 \mathcal{M}_{0}$ :

$$
\mathbf{E}_{2 \omega_{0}}=\mathcal{E}_{2 \omega_{0}}^{(0)}+\mathcal{E}_{2 \omega_{0}}^{(1)}+\cdots
$$

where the first two terms are

$$
\mathcal{E}_{2 \omega_{0}}^{(0,1)}=-\nabla \Phi_{2 \omega_{0}}^{(0,1)}
$$

and the potential functions $\Phi_{2 \omega_{0}}^{(0,1)}$ are the solutions of the Laplace equation:

$$
\nabla^{2} \Phi_{2 \omega_{0}}^{(0,1)}=0 \quad(r<a, r>a) .
$$

At the boundary $r=a$, the tangential components of electric fields are continuous while the normal components of the electric displacement vectors $\varepsilon(\mathbf{r})\left(-\nabla \Phi_{2 \omega_{0}}^{(0,1)}\right)$ experience discontinuities due to the surface charge density $\rho_{2 \omega_{0}}^{(0,1)}=$ $\nabla_{s} \cdot\left(\mathbf{j}_{2 \omega_{0}}^{(0,1)}+\sigma_{2} \mathcal{E}_{2 \omega_{0 \tau}}^{(0,1)}\right) / i 2 \omega_{0}$, where $\sigma_{2} \equiv \sigma\left(2 \omega_{0}\right)$. To find field distribution, we need to satisfy these boundary conditions and we expand $\Phi_{2 \omega_{0}}^{(0,1)}(\mathbf{r})$ in spherical harmonics. $\nabla_{s} \cdot \mathbf{j}_{2 \omega_{0}}^{(0)}$ is proportional to $(1+3 \cos 2 \theta)$, and thus, the respective potential relative to a symmetric locally excited quadrupole is expressed as follows:

$$
\Phi_{2 \omega_{0}}^{(0)}=\frac{(1+3 \cos 2 \theta)}{4} Q_{2 \omega_{0}}^{(0)} \begin{cases}\frac{1}{r^{3}}, & r>a \\ \frac{r^{2}}{a^{5}}, & r<a,\end{cases}
$$

where the quadrupole moment component

$$
Q_{2 \omega_{0}}^{(0)}=\frac{3}{2} \frac{e^{3} v_{F}^{2}}{\hbar^{2} \omega_{0}^{4}\left(3+2 \varepsilon_{p}-3 \Theta_{2}\right)} a^{2} E_{d}^{2},
$$

where $\Theta_{2}=4 \pi \sigma_{2} / i \omega_{0} a$.

We would like to note that a similar analysis can be performed for metal nanoparticles using a free-electron hydrodynamic model. Generally, the potential functions $\Phi_{2 \omega_{0}}^{(0)}$ and $\Phi_{2 \omega_{0}}^{(1)} \sim \cos \tilde{\theta} \equiv \sin \theta \sin \varphi$ for $r>a$ would coincide with the potentials of the quadrupole, which is symmetric with respect to the $z$ axis and has a diagonal tensor of the quadrupole moment

$$
\hat{Q}_{2 \omega_{0}}^{(0)}=Q_{2 \omega_{0}}^{(0)}\left(\begin{array}{rrr}
-1 & 0 & 0 \\
0 & -1 & 0 \\
0 & 0 & 2
\end{array}\right)
$$

and of the dipole with the dipole moment

$$
\mathcal{P}_{2 \omega_{0}}^{(1)}=\mathcal{P}_{2 \omega_{0}}^{(1)} \mathbf{y}_{0},
$$

which is directed along the wave vector of the incident wave. The radiation intensities (powers) of the quadrupole $\hat{Q}_{2 \omega_{0}}$ and the dipole $\mathcal{P}_{2 \omega_{0}}$ moments given by [54]

$$
\begin{aligned}
P_{\Sigma}^{(\text {quad })} & =\frac{c\left(2 k_{0}\right)^{6}}{60}\left|Q_{2 \omega_{0}}^{(0)}\right|^{2}, \\
P_{\Sigma}^{(\text {dip })} & =\frac{c\left(2 k_{0}\right)^{4}}{3}\left|\mathcal{P}_{2 \omega_{0}}^{(1)}\right|^{2}
\end{aligned}
$$
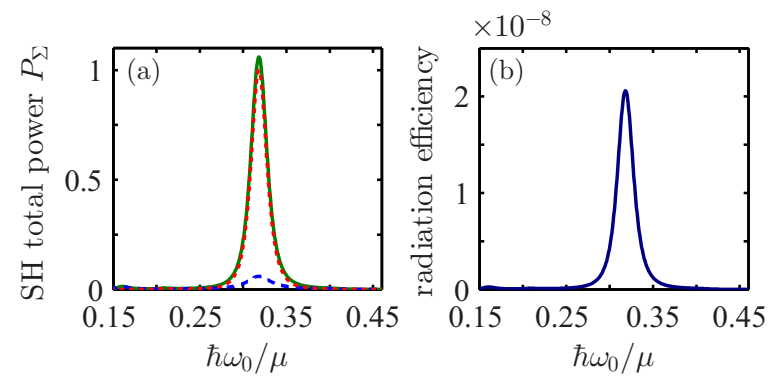

FIG. 2. (Color online) (a) Radiated power and (b) secondharmonic conversion efficiency as functions of frequency calculated for a nanoparticle of radius $a=106 \mathrm{~nm}, \varepsilon_{p}=2.1, N=1, \mu=$ $0.25 \mathrm{eV}, \tau_{\text {intra }}=0.08 \mathrm{ps}$, and incident intensity $10^{8} \mathrm{~W} / \mathrm{cm}^{2}$. In (a) the green solid line shows the total radiated power, and the blue dashed and red dotted lines refer to the dipole and quadrupole contributions, respectively. Powers are normalized to the quadrupole maximum value.

have the same order of smallness. The radiation pattern of the quadrupole is symmetric with respect to the $z$ axis, along which the electric field in the incident wave is oriented and has maxima at $\theta=\pi / 4,3 \pi / 4$. The dipole radiation is symmetric with respect to the wave vector of the incident wave, parallel to the $y$ axis, and is maximum in the $y=0$ plane.

In our case of a graphene sphere, the dipole moment associated with the current $\mathbf{j}_{2 \omega_{0}}^{(1)}$ is given by the expression

$$
\begin{aligned}
\mathcal{P}_{2 \omega_{0}}^{(1)}= & \frac{3}{4} \frac{i e^{3} v_{F}^{2}}{\hbar^{2} \omega_{0}^{4}\left(3 / 2+\varepsilon_{p}-3 \Theta_{1}\right)} \\
& \times \frac{5 / 4+\varepsilon_{p}-3 \Theta_{1}}{2+\varepsilon_{p}-\Theta_{2}} k_{0} a^{2} E_{d} E_{0} .
\end{aligned}
$$

As follows from Eqs. (20a), (25), and (29), the resonant enhancement in the second-harmonic ( $\mathrm{SH})$ scattering occurs near the frequencies of localized plasmons. The total radiated $\mathrm{SH}$ power possesses a pronounced peak at the frequency of the one-photon dipolar resonance where the radiation pattern appears to be predominantly quadrupolar, as shown in Fig. 2(a).

We note here that the scattering object containing graphene benefits from its tunability. In our problem, there is a large number of parameters that can be adjusted for controlling the effect. In particular, one can choose the operating frequency $\omega_{0}$, the chemical potential of graphene $\mu$, the particle radius $a$, the number of graphene layers in the wrapping $N$, the dielectric permittivity of the particle $\varepsilon_{p}$, and the relaxation time $\tau_{\text {intra }}$, which is responsible for the broadening of the resonant peaks.

The SH signal from a single nanoparticle is rather weak. To quantify the conversion efficiency, we introduce the SHG efficiency for a single particle $\eta$ defined as the ratio of the total $\mathrm{SH}$ radiated power to the energy flux of the fundamental wave through the physical area of the particle $s=\pi a^{2}$, $\eta=P_{\Sigma} /\left[(c / 8 \pi)\left|E_{0}\right|^{2} \pi a^{2}\right]$. Dependence of the efficiency on frequency clearly shows resonance [see Fig. 2(b)], and in the maximum it reaches the value of $\sim 2 \times 10^{-8}$. 


\section{CONTROL OF RADIATION PATTERNS BY INHOMOGENEOUS EXTERNAL FIELDS}

Now we consider the case when a graphene-wrapped nanoparticle is placed in a weakly inhomogeneous quasistatic external field, which is axially symmetric with respect to the $z$ axis (see schematics in Fig. 3). Such field distribution can be realized in two counterpropagating Bessel beams forming a standing wave. Similar field profiles can be realized if we place our nanoparticle near a larger particle, e.g., an elongated nanoparticle or near a tip of an atomic force microscope, positioned in the maximum of the standing wave created by two counterpropagating plane waves.

Using the Taylor series expansion around the center of the particle, we introduce the electric potential of the external field in the form which satisfies the Laplace equation:

$$
\Phi_{\omega_{0}}^{(\mathrm{ext})}=-E_{0} r \cos \theta-\frac{1}{8} \frac{\partial E_{0}}{\partial z} r^{2}(1+3 \cos 2 \theta)+\cdots,
$$

where $\frac{\partial E_{0}}{\partial z} \equiv \gamma$ is a tunable parameter characterizing variation of the field along the $z$ axis. Next, we follow the procedure described above, expand the fields, and apply the boundary conditions.

In the previous sections we considered the linear dipole described by the first term in Eq. (30) and associated quadrupole at the double frequency. The resonant enhancement in this case due to surface plasmon resonances is expected near the frequencies where $\varepsilon_{p}=-2+8 \pi \operatorname{Im} \sigma\left(\omega_{0}\right) / \omega_{0} a$ and $\varepsilon_{p}=-3 / 2+6 \pi \operatorname{Im} \sigma\left(2 \omega_{0}\right) / \omega_{0} a$, i.e., where the real parts of the corresponding denominators vanish. However, as we show in Fig. 4, the one-photon dipolar plasmon resonance is much more pronounced than the two-photon quadrupolar.

The field inhomogeneity given by the second term in Eq. (30) determines a linear potential

$$
\Phi_{\omega_{0}}^{(1)}=\frac{(1+3 \cos 2 \theta)}{4} Q_{\omega_{0}}^{(1)} \begin{cases}\frac{1}{r^{3}}, & r>a \\ \frac{r^{2}}{a^{5}}, & r<a\end{cases}
$$

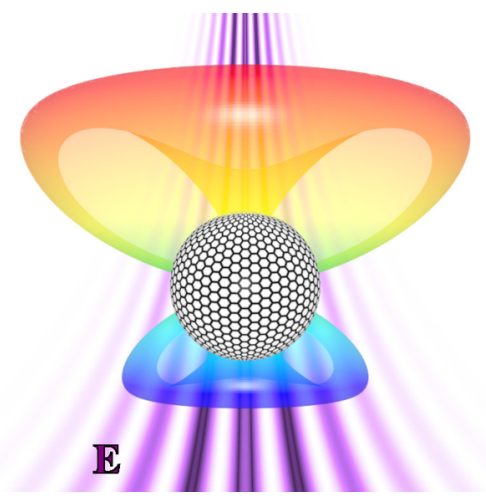

FIG. 3. (Color online) Schematic view of a graphene-wrapped nanoparticle placed into an axially symmetric slightly inhomogeneous external field. The SH radiation is predominantly directed into the upper half-space.

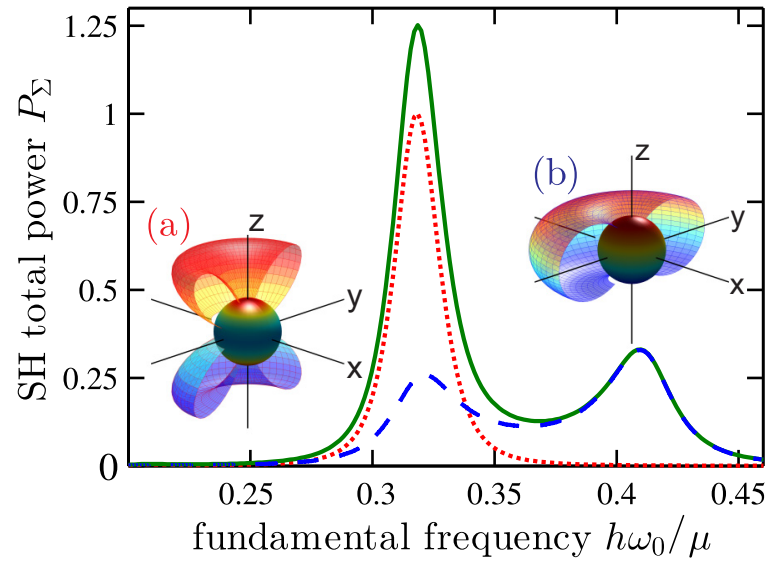

FIG. 4. (Color online) Spectra of the radiated powers of the SH quadrupole (red dotted curve), SH dipole (blue dashed curve), and total SH emission (green solid curve), calculated for a nanoparticle of radius $a=106 \mathrm{~nm}, \varepsilon_{p}=2.1, N=1, \mu=0.25 \mathrm{eV}, \tau_{\text {intra }}=0.08 \mathrm{ps}$, and $\bar{\gamma}=0.5$. Powers are normalized to the pure quadrupole maximum value. Insets show far-field radiation patterns and surface charge distributions of the pure (a) SH quadrupole $(\bar{\gamma}=0)$, and (b) $\mathrm{SH}$ dipole, which is excited predominantly near the higher-frequency resonance.

that can be attributed to a symmetric quadrupole

$$
\hat{Q}_{\omega_{0}}^{(1)}=Q_{\omega_{0}}^{(1)}\left(\begin{array}{rrr}
-1 & 0 & 0 \\
0 & -1 & 0 \\
0 & 0 & 2
\end{array}\right)
$$

with the amplitude

$$
Q_{\omega_{0}}^{(1)}=\frac{\varepsilon_{p}-1-3 \Theta_{1}}{2 \varepsilon_{p}+3-6 \Theta_{1}} \gamma a^{5} .
$$

Now we calculate the induced second-harmonic current

$$
\mathbf{j}_{2 \omega_{0}}^{(1)}=-i \frac{e^{3}}{\pi \hbar^{2} \omega_{0}^{3}} \frac{v_{F}^{2}}{a} E_{d} E_{q}^{(\mathrm{ext})} \boldsymbol{\theta}_{0} \frac{1}{32}(3 \sin 3 \theta-\sin \theta),
$$

where the quadrupole amplitude coefficient is

$$
E_{q}^{(\mathrm{ext})}=-\frac{15}{4} \frac{\gamma a}{2 \varepsilon_{p}+3-6 \Theta_{1}},
$$

and taking the surface divergence $\nabla_{s} \cdot \mathbf{j}_{2 \omega_{0}}^{(1)} \sim[-3.2 \cos \theta+$ 9.6(5 $\left.\left.\cos ^{3} \theta-3 \cos \theta\right)\right]$ containing the leading term proportional to $\cos \theta$ we obtain the respective potential

$$
\Phi_{2 \omega_{0}}^{(1)}=\mathcal{P}_{2 \omega_{0}}^{(1)} \cos \theta \begin{cases}\frac{r}{a^{3}}, & |\mathbf{r}|<a \\ \frac{1}{r^{2}}, & |\mathbf{r}|>a\end{cases}
$$

that corresponds to a dipole oriented along the $z$ axis

$$
\mathcal{P}_{2 \omega_{0}}^{(1)}=\mathcal{P}_{2 \omega_{0}}^{(1)} \mathbf{z}_{0},
$$

with a dipole moment

$$
\mathcal{P}_{2 \omega_{0}}^{(1)}=\frac{3.2}{16} \frac{e^{3} v_{F}^{2}}{\hbar^{2} \omega_{0}^{4}\left(\varepsilon_{p}+2-\Theta_{2}\right)} a E_{d} E_{q}^{(\mathrm{ext})} .
$$

Remarkably, this $\mathrm{SH}$ dipole is aligned along the direction of the electric field of fundamental frequency, similar to the dipole 

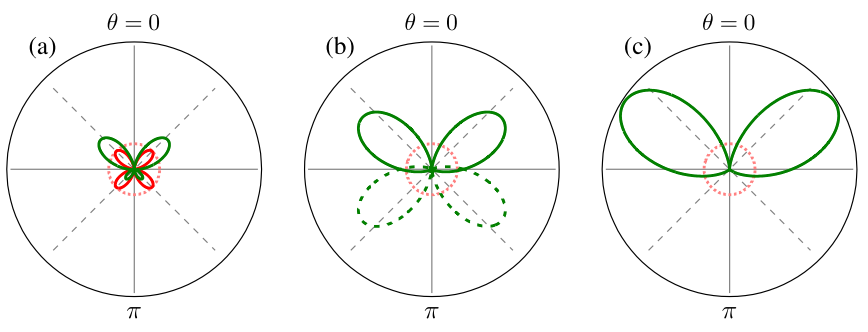

FIG. 5. (Color online) $\mathrm{SH}$ radiation patterns shown as polar $\theta$ diagrams (green solid lines) calculated at $\hbar \omega_{0}=0.318 \mu$ for the parameters of Fig. $4\left(k_{0} a \approx 0.06\right)$ and different values of $\bar{\gamma}$ : (a) $\bar{\gamma}=0.5$, (b) $\bar{\gamma}=1.25$, and the reverse at $\bar{\gamma}=-1.25$ (dashed green line), and (c) $\bar{\gamma}=1.75$. The inner circle in all the plots corresponds to the same maximum intensity of the pure SH quadrupole. For reference, a four-lobed pattern of a radiating $\mathrm{SH}$ quadrupole source $(\bar{\gamma}=0)$ is also depicted in panel (a).

induced by linear scattering, and it is in sharp contrast with the conventional nonlinear dipole arising from the retardation.

The function of the dipolar radiated power plotted in Fig. 4 exhibits two peaks close to the frequencies estimated from $\varepsilon_{p}=-2+8 \pi \operatorname{Im} \sigma\left(\omega_{0}\right) / \omega_{0} a$ and $\varepsilon_{p}=$ $-3 / 2+12 \pi \operatorname{Im} \sigma\left(\omega_{0}\right) / \omega_{0} a$, dominating the two-photon dipole resonance at the lower frequency around $\varepsilon_{p}=-2+$ $4 \pi \operatorname{Im} \sigma\left(2 \omega_{0}\right) / \omega_{0} a$.

The radiation pattern at $2 \omega_{0}$ being determined now by both dipolar and quadrupolar contributions can be varied. We illustrate this aspect with examples of the radiation pattern transformations in the far field at frequency $\hbar \omega_{0}=0.318 \mu$ close to the peak SHG enhancement. The angular distributions of the $\mathrm{SH}$ radiation are shown in Fig. 5 as polar plots for different values of a dimensionless parameter $\bar{\gamma}=\bar{\gamma} E_{0} / a$. These diagrams show that the $\mathrm{SH}$ radiation predominantly occurs in the direction of the stronger field at fundamental frequency. Note, near the points where $E_{0}$ vanishes, for example, near the nodes of the standing Bessel wave, the value of $\bar{\gamma}$ can be arbitrarily large, although the total radiation power is obviously decreased. Importantly, the radiation pattern remains axially symmetric with respect to the $z$ axis but due to the phase relations at some value of $\bar{\gamma}$ two lower lobes almost disappear and the $\mathrm{SH}$ source effectively emits into the upper half-space as shown in Fig. 5(b). This is in sharp contrast to the conventional multipolar interaction for small spherical nanoparticles, placed in the field of the plane monochromatic wave (see Sec. IV), where the resonant enhancement close to the distinctive localized plasmon resonances is never accompanied by strongly asymmetric radiation patterns since the dipole and quadrupole fields are out of phase, and hence do not interfere (see, e.g., Ref. [17]). We note that the directivity can be changed to the opposite by changing the sign of $\bar{\gamma}$ (for example, moving the particle along the standing wave). Subsequent increase of the $\bar{\gamma}$ leads to the gradual transformation of the radiation pattern into dipolelike [see an intermediate step in Fig. 5(c)].

\section{CONCLUSION}

We have studied the second-harmonic generation by a nanoparticle wrapped into graphene. We have derived the analytical model and analyzed efficiency and radiation directionality of the second-harmonic radiation. We have demonstrated the possibility to control the radiation pattern with an inhomogeneous external field that breaks a balance between the dipole and quadrupole radiation contributions. For the typical parameters, we have predicted that the second harmonic is predominantly radiated in the half-space with stronger external electric field.

\section{ACKNOWLEDGMENTS}

The authors thank Sergey A. Mikhailov for useful discussions of the results, and Lukas Novotny for his interest in this project. This work was supported by the Australian Research Council. A.I.S. acknowledges support from RFBR, Grant No. 13-02-00881.
[1] F. Brown, R. E. Parks, and A. M. Sleeper, Phys. Rev. Lett. 14, 1029 (1965).

[2] J. E. Sipe, V. C. Y. So, M. Fukui, and G. I. Stegeman, Phys. Rev. B 21, 4389 (1980).

[3] J. E. Sipe and G. I. Stegeman, Surface Polaritons: Electromagnetic Waves at Surfaces and Interfaces (North-Holland, Amsterdam, 1982).

[4] F. X. Wang, F. J. Rodriguez, W. M. Albers, R. Ahorinta, J. E. Sipe, and M. Kauranen, Phys. Rev. B 80, 233402 (2009).

[5] F. Hache, D. Ricard, and C. Flytzanis, J. Opt. Soc. Am. B 3, 1647 (1986).

[6] X. M. Hua and J. I. Gersten, Phys. Rev. B 33, 3756 (1986).

[7] J. Rudnick and E. A. Stern, Phys. Rev. B 4, 4274 (1971).

[8] P. Guyot-Sionnest, W. Chen, and Y. R. Shen, Phys. Rev. B 33, 8254 (1986).

[9] P. Guyot-Sionnest and Y. R. Shen, Phys. Rev. B 38, 7985 (1988).

[10] M. Kauranen and A. V. Zayats, Nat. Photonics 6, 737 (2012).
[11] A. Boltasseva and V. M. Shalaev, Metamaterials 2, 1 (2008).

[12] G. S. Agarwal and S. S. Jha, Solid State Commun. 41, 499 (1982).

[13] J. I. Dadap, J. Shan, K. B. Eisenthal, and T. F. Heinz, Phys. Rev. Lett. 83, 4045 (1999).

[14] E. V. Makeev and S. E. Skipetrov, Opt. Commun. 224, 139 (2003).

[15] W. L. Mochan, J. A. Maytorena, B. S. Mendoza, and V. L. Brudny, Phys. Rev. B 68, 085318 (2003).

[16] C. I. Valencia, E. R. Mendez, and B. S. Mendoza, J. Opt. Soc. Am. B 20, 2150 (2003).

[17] J. I. Dadap, J. Shan, and T. F. Heinz, J. Opt. Soc. Am. B 21, 1328 (2004).

[18] K. A. O’Donnell and R. Torre, New J. Phys. 7, 154 (2005).

[19] G. Bachelier, I. Russier-Antoine, E. Benichou, C. Jonin, and P. F. Brevet, J. Opt. Soc. Am. B 25, 955 (2008).

[20] G. Bachelier, J. Butet, I. Russier-Antoine, C. Jonin, E. Benichou, and P.-F. Brevet, Phys. Rev. B 82, 235403 (2010). 
[21] A. G. F. deBeer and S. Roke, Phys. Rev. B 79, 155420 (2009).

[22] P. Ginzburg, A. Krasavin, Y. Sonnefraud, A. Murphy, R. J. Pollard, S. A. Maier, and A. V. Zayats, Phys. Rev. B 86, 085422 (2012).

[23] S. G. Rodrigo, H. Harutyunyan, and L. Novotny, Phys. Rev. Lett. 110, 177405 (2013).

[24] M. Jablan, H. Buljan, and M. Soljacic, Phys. Rev. B 80, 245435 (2009).

[25] A. N. Grigorenko, M. Polini, and K. S. Novoselov, Nat. Photonics 6, 749 (2012).

[26] Q. Q. Bao and K. P. Loh, ACS Nano 6, 3677 (2012).

[27] M. Jablan, M. Soljacic, and H. Buljan, Proc. IEEE 101, 1689 (2013).

[28] X. Luo, T. Qiu, W. Lu, and Z. Ni, Mater. Sci. Eng., R 74, 351 (2013).

[29] Z. Fei, A. S. Rodin, G. O. Andreev, W. Bao, A. S. McLeod, M. Wagner, L. M. Zhang, Z. Zhao, G. Dominguez, M. Thiemens, M. M. Fogler, A. H. Castro-Neto, C. N. Lau, F. Keilmann, and D. N. Basov, Nature (London) 487, 82 (2012).

[30] J. Chen, M. Badioli, P. Alonso-Gonzales, S. Thongrattanasiri, F. Huth, J. Osmond, M. Spasenovic, A. Centeno, A. Pesquera, P. Godignon, A. Z. Elorza, N. Camara, F. J. G. de Abajo, R. Hillenbrand, and F. H. L. Koppens, Nature (London) 487, 77 (2012).

[31] S. A. Mikhailov, Europhys. Lett. 79, 27002 (2007).

[32] S. A. Mikhailov and K. Ziegler, J. Phys.: Condens. Matter 20, 384204 (2008).

[33] X. Yao and A. Belyanin, Phys. Rev. Lett. 108, 255503 (2012).

[34] E. G. Mishchenko, Phys. Rev. Lett. 103, 246802 (2009).

[35] M. M. Glazov and S. D. Ganichev, Phys. Rep. 535, 101 (2014).

[36] M. L. Nesterov, J. Bravo-Abad, A. Yu. Nikitin, F. J. GarciaVidal, and L. Martin-Moreno, Laser Photonics Rev. 7, L7 (2013).

[37] D. A. Smirnova, A. V. Gorbach, I. V. Iorsh, I. V. Shadrivov, and Y. S. Kivshar, Phys. Rev. B 88, 045443 (2013).
[38] D. A. Smirnova, I. V. Shadrivov, A. I. Smirnov, and Y. S. Kivshar, Laser Photonics Rev. 8, 291 (2014).

[39] J. W. Ko, S.-W. Kim, J. Hong, K. Kang, and C. B. Park, Green Chem. 14, 2391 (2012).

[40] J. S. Lee, K. H. You, and C. B. Park, Adv. Mater. 24, 1084 (2012).

[41] S. Sheng, L. Zhang, and G. Chen, Electrochem. Commun. 24, 13 (2012).

[42] M. Farhat, C. Rockstuh, and H. Bagci, Opt. Express 21, 12592 (2013).

[43] P. Y. Chen and A. Alu, ACS Nano 5, 5855 (2011).

[44] P. Y. Chen, J. Soric, Y. R. Padooru, H. M. Bernety, A. B. Yakovlev, and A. Alu, New J. Phys. 15, 123029 (2013).

[45] L. A. Falkovsky and S. S. Pershoguba, Phys. Rev. B 76, 153410 (2007).

[46] T. Stauber, N. M. R. Peres, and A. K. Geim, Phys. Rev. B 78, 085432 (2008)

[47] F. T. Vasko and V. Ryzhii, Phys. Rev. B 76, 233404 (2007).

[48] L. A. Falkovsky and A. A. Varlamov, Eur. Phys. J. B 56, 281 (2007).

[49] A. A. Dubinov, V. Ya. Aleshkin, V. Mitin, T. Otsuji, and V. Ryzhii, J. Phys.: Condens. Matter 23, 145302 (2011).

[50] G. W. Hanson, J. Appl. Phys. 104, 084314 (2008).

[51] M. Nakamura and L. Hirasawa, Phys. Rev. B 77, 045429 (2008).

[52] M. Orlita and M. Potemski, Semicond. Sci. Technol. 25, 063001 (2010).

[53] D. A. Smirnova, I. V. Iorsh, I. V. Shadrivov, and Y. S. Kivshar, JETP Lett. 99, 456 (2014).

[54] J. Jackson, Classical Electrodynamics (Mir, Moskow, 1965).

[55] C. F. Bohren and D. R. Huffman, Absorption and Scattering of Light by Small Particles (Wiley, New York, 1983).

[56] L. Novotny and B. Hecht, Principles of Nano-Optics (Cambridge University Press, New York, 2007).

[57] M. M. Glazov, JETP Lett. 93, 366 (2011).

[58] S. A. Mikhailov, Phys. Rev. B 84, 045432 (2011). 